COMMENT. The intramuscular fatigability and recovery following sustained maximum voluntary contraction was similar in dystrophic muscles and controls, but patients with DMD had less central fatigue, possibly explained by longer training sessions and greater familiarity with the exercise.

\title{
PELIZAEUS-MERZBACHER DISEASE AND HYPOTONIA
}

Two brothers with Pelizaeus-Merzbacher disease presenting with neonatal hypotonia and hyporeflexia are reported from the Tuft's University School of Medicine, and Massachusetts General Hospital, Boston, and the EKS Center for Mental Retardation, Waltham, MA. Patient 1 had Apgar scores of 0 at 1 minute and 3 at 5 minutes. The neonatal exam showed signs of spinal muscular atrophy: poor suck and swallow, tongue fasciculations, reduced muscle bulk and strength, severe hypotonia, absent deep tendon reflexes, and normal sensation. An older brother had a similar disease. Parents were not related. Nystagmoid eye movements were present shortly after birth and disappeared before 1 year. Optic atrophy and dystonic arm movements developed after several months. MRI showed diffuse hypomyelination. EMG demonstrated fibrillations, high-amplitude potentials, and acute denervation. Muscle biopsy was normal. The diagnosis of Pelizaeus-Merzbacher disease was confirmed by proteolipid protein genetic studies. (Kaye EM et al. PelizaeusMerzbacher disease presenting as spinal muscular atrophy: Clinical and molecular studies. Ann Neurol Dec 1994;36:916-919). (Respond: Dr Kaye, Division of Pediatric Neurology, Floating Hospital for Children, Tuft's University School of Medicine, Boston, MA 02111).

COMMENT. Pelizaeus-Merzbacher disease should be included in the differential diagnosis of limp infant syndrome and as a cause of infantile spinal muscular atrophy.

\section{NEUROCUTANEOUS SYNDROMES}

\section{LEUKOENCEPHALOPATHY AND KERATODERMA}

A new familial neurocutaneous syndrome consisting of palmoplantar keratoderma (PPK) and adult-onset leukoencephalopathy is reported in four siblings from Hadassah University Hospital, Jerusalem, Israel. The proband, a 41-year-old woman, had a progressive gait disorder and cognitive impairment of 3 years' duration. Skin abnormalities had been present from early childhood: thick hyperkeratotic skin with numerous papules, most prominent over the palmar and plantar areas. Neurologic exam revealed cognitive impairments, generalized weakness with symmetric hyperreflexia, hypertonia, and Babinski signs. CSF protein was $1.18 \mathrm{~g} / \mathrm{L}$. MRI showed periventricular hyperintensity of white matter, brain atrophy, and thinning of the corpus callosum. Arylsulfatase A pseudodeficiency carrier state was identified by molecular analysis. Sural nerve biopsy showed loss of myelinated fibers. Bone X-rays showed osteochondritis dissecans of the talus. (Lossos A et al. Hereditary leukoencephalopathy and palmoplantar keratoderma: A new disorder with increased skin collagen content. Neurology Feb 1995;45:331-337). (Reprints: Dr A Lossos, Dept Neurol, Hadassah Univ Hosp, Jerusalem 91120, Israel.

COMMENT. Neurologic complications of PPK are rare and late in onset. 